\title{
RibAlign: a software tool and database for eubacterial phylogeny based on concatenated ribosomal protein subunits Hanno Teeling ${ }^{* 1}$ and Frank Oliver Gloeckner ${ }^{1,2}$
}

Address: ${ }^{1}$ Microbial Genomics Group, Max Planck Institute for Marine Microbiology, D-28359 Bremen, Germany and ${ }^{2}$ International University Bremen, D-28759 Bremen, Germany

Email: Hanno Teeling* - hteeling@mpi-bremen.de; Frank Oliver Gloeckner -f.gloeckner@iu-bremen.de

* Corresponding author

Published: 13 February 2006

BMC Bioinformatics2006, 7:66 doi:10.1|86/147|-2105-7-66

This article is available from: http://www.biomedcentral.com/I47I-2105/7/66

(C) 2006Teeling and Gloeckner; licensee BioMed Central Ltd.

This is an Open Access article distributed under the terms of the Creative Commons Attribution License (http://creativecommons.org/licenses/by/2.0), which permits unrestricted use, distribution, and reproduction in any medium, provided the original work is properly cited.
Received: 10 June 2005

Accepted: I3 February 2006

\begin{abstract}
Background: Until today, analysis of I6S ribosomal RNA (rRNA) sequences has been the de-facto gold standard for the assessment of phylogenetic relationships among prokaryotes. However, the branching order of the individual phlya is not well-resolved in $16 \mathrm{~S}$ rRNA-based trees. In search of an improvement, new phylogenetic methods have been developed alongside with the growing availability of complete genome sequences. Unfortunately, only a few genes in prokaryotic genomes qualify as universal phylogenetic markers and almost all of them have a lower information content than the 16S rRNA gene. Therefore, emphasis has been placed on methods that are based on multiple genes or even entire genomes. The concatenation of ribosomal protein sequences is one method which has been ascribed an improved resolution. Since there is neither a comprehensive database for ribosomal protein sequences nor a tool that assists in sequence retrieval and generation of respective input files for phylogenetic reconstruction programs, RibAlign has been developed to fill this gap.
\end{abstract}

Results: RibAlign serves two purposes: First, it provides a fast and scalable database that has been specifically adapted to eubacterial ribosomal protein sequences and second, it provides sophisticated import and export capabilities. This includes semi-automatic extraction of ribosomal protein sequences from whole-genome GenBank and FASTA files as well as exporting aligned, concatenated and filtered sequence files that can directly be used in conjunction with the PHYLIP and MrBayes phylogenetic reconstruction programs.

Conclusion: Up to now, phylogeny based on concatenated ribosomal protein sequences is hampered by the limited set of sequenced genomes and high computational requirements. However, hundreds of full and draft genome sequencing projects are on the way, and advances in cluster-computing and algorithms make phylogenetic reconstructions feasible even with large alignments of concatenated marker genes. RibAlign is a first step in this direction and may be particularly interesting to scientists involved in whole genome sequencing of representatives of new or sparsely studied eubacterial phyla. RibAlign is available at http://www.megx.net/ribalign 


\section{Background}

Analysis of 16S ribosomal rRNA (rRNA) sequences is currently the de-facto gold standard for the assessment of phylogenetic relationships among prokaryotes. There are various reasons that have made the 16S rRNA gene the first choice as a phylogenetic marker, such as the presence of positions with different evolutionary rates, its universal occurrence within prokaryotes, its reasonable information content, a length that was suitable for complete sequencing when the technique started, knowledge about its secondary structure that helps with alignments and finally the presence of a comprehensive database of more than hundred thousand sequences [1]. With ARB [2], there is also a well-curated 16S rRNA database with a curated alignment and a program suite for phylogenetic reconstructions available that has gained broad acceptance among scientists worldwide.

Despite this success, trees based on 16S rRNA sequences lack resolution when it comes to elucidating the branching order of individual phyla [3]. This limits our understanding of early evolutionary splits within the prokaryotes and the degree of relatedness among individual phyla, of which some have been proposed to build super-clusters $[4,5]$. These issues still are matters of sometimes heated debates $[6,7]$. It is possible that particularly early evolution can never be fully determined because an early evolutionary boundary limits the attainable resolution. The cause for this boundary might be either (a) methodological and caused by the limited information content (i.e. mutational saturation) of single marker genes, or (b) fundamental and caused by extensive lateral gene transfer (LGT) among early prokaryotes [8-10].

Before the genomic revolution, it had been anticipated that the wealth of information from entire genomes would lead to a refined view on the tree of life. Consequently, the ever-growing availability of complete genome sequences has propelled the development of new phylogenetic methods. Some of these methods exploit information from entire genomes whereas others use only a subset. Examples are super-tree approaches that combine individual trees $[11,12]$, methods based on comparisons of genes between organisms (shared gene content [13-16], shared gene order [15], similarities of protein folds and domains $[17,18])$, methods based on intrinsic DNA-signatures (e.g. skewed oligonucleotide distributions) [19] and concatenations of marker genes [4,20-27].

It is one of the big disillusions of the post-genomic era, that most of these methods fail to provide an advantage in resolution over $16 \mathrm{~S}$ rRNA-based trees [5]. Instead, comparative genomics revealed an extent of LGT that seriously questions the applicability of the eukaryotic species concept to the world of the prokaryotes. As a result, today the tree of life must be regarded as a complex network of vertical and horizontal inheritance. The extent to which tree reconstruction is affected by LGT is still a matter of debate [28]. It has been argued that a subset of the genes, including those encoding (most) ribosomal proteins, are less likely to undergo LGT and that for these core genes a phylogeny can be reliably inferred [28-31]. Whether such a stable genetic core really exists is hard to prove and hence discussed controversially $[8,11,12]$. Its existence is supported by the fact that phylogenetic analysis of alleged core genes in general support the 16S-derived three domain concept and mostly also correlate with $16 \mathrm{~S}$ rRNA analysis in detail - a congruence that is notably absent form most non-core genes [30]. From the core genes, ribosomal proteins are of particular interest because their tight interactions with the $16 \mathrm{~S}$ and other rRNAs suggests co-evolution of these molecules. Moreover, concatenation of ribosomal protein sequences is one of the few methods that has been ascribed an enhanced resolution [5]. This is also reflected in a variety of publications on phylogenetic reconstructions that are based on this method $[4,20$ $25,27]$.

As of this writing (May 2005), 224 completely sequenced eubacterial genomes are available to the public. Hence, the data set available for comparison of ribosomal protein sequences is sparse when compared to the vast amount of available 16S rRNA sequences. On the other hand, most of the known phyla have been covered by at least one sequenced representative, and the gaps are being filled quickly. In addition, most draft genome sequences contain most if not all of the ribosomal proteins, so that the method is not necessarily restricted to fully closed genomes.

\section{Implementation}

RibAlign has been implemented in a fully object-oriented manner with REALbasic [32] and uses the high-performance Valentina object-relational database engine [33] to store sequences and related information.

New sequences can be imported from whole-genome GenBank or FASTA files. An automated screening of the annotated gene descriptions and gene names assists in the extraction of the ribosomal proteins before writing them to the database. The database and importer has currently been designed for the extraction and storage of sequences from Eubacteria, but future releases of RibAlign might include archaeal ribosomal protein sequences as well.

RibAlign can not only export sequences to plain FASTA format, but also has a complete built-in pipeline for generating processed input files for the PHYLIP [34] and MrBayes [35,36] phylogenetic reconstruction programs. This pipeline comprises exporting dedicated multi- 


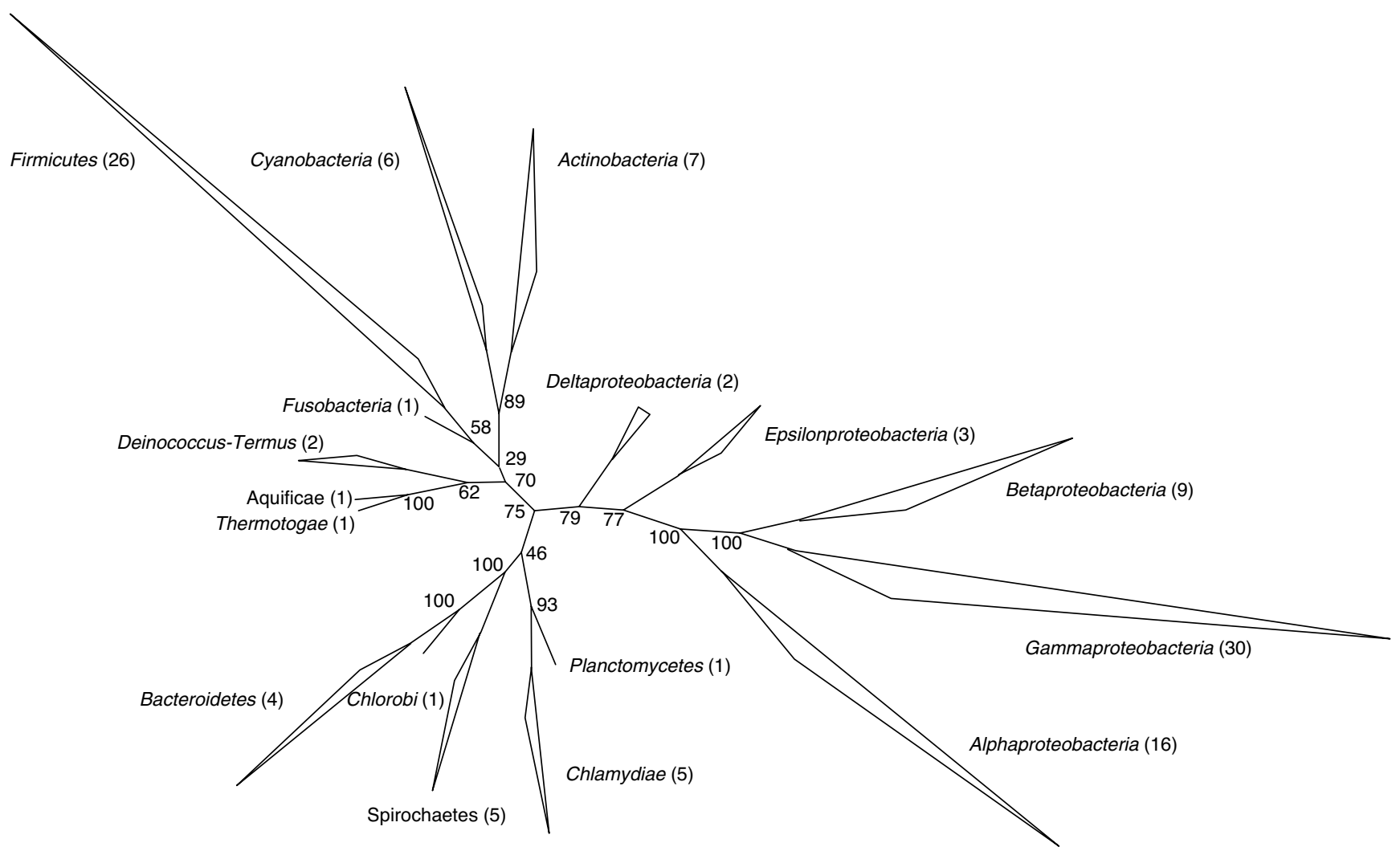

Figure I

Unrooted maximum-likelihood tree based on concatenated ribosomal proteins for I 20 eubacterial species. The following 37 sequences were concatenated: RpIABCDFIJKLMNOPQRSTUVW, RpmA, RpsBCDEFGHIKLMOQRST, filter by a 40\% positional conservation filter resulting in 5182 amino acid positions. The tree was calculated from this alignment using the ProML program of the PLYLIPackage (settings: best tree search mode; JTT model of amino acid substitution; one category of sites with a constant evolutionary rate; no weights; rough-type of analysis with global rearrangements). Bootstrapping was carried out with 100 replicates. Numbers in parentheses refer to the numbers of species that make up the respective branch.

headed FASTA files for a selectable subset of ribosomal proteins, alignment of the exported sequences independently for each gene, concatenation of the individual alignments into a single alignment, filtering of the lessconserved positions according to an adjustable threshold and finally conversion to PHYLIP or NEXUS format.

RibAlign does not implement its own alignment algorithm but instead uses the MAFFT program [37], which can generate high-quality alignments with good speed even when used with larger sets of sequences. MAFFT is not part of RibAlign's distribution and thus has to be obtained and installed separately [38].

RibAlign comprises a searchable, tutorial-like online help that provides detailed information on all of the program's features.

We expect the implementation of RibAlign and the underlying database to perform nicely with the upcoming flood of genome sequences, since it has been tested with 10,000 artificial entries. The current release of RibAlign requires Mac OS X and as of this writing, no decisions on possible ports to other platforms have been made. Contributions concerning this matter are welcome.

RibAlign is freely available for academic applications and can be downloaded from its website [39], which also provides screenshots of RibAlign's user interface.

\section{Results and discussion}

\section{Construction and quality of the RibAlign data set}

RibAlign is bundled with an example database (RibAlignDB) that contains the ribosomal protein sequences of 184 of the publicly available complete eubacterial genome sequences. This data set has been generated by importing the respective GenBank files, followed by some manual curation. The latter comprised shifting N-termini of sequences, deleting false paralogs, cross-checking of dubious annotations by InterPro [40] searches and in 
some cases re-annotation of falsely annotated ribosomal proteins. Despite these efforts, RibAlign's data set can by no means be regarded as well-curated. Like all genome annotations, it does contain errors. Thus, data should be checked carefully prior to using it for phylogenetic reconstructions. Archaea are currently not included, since (a) so far only 22 complete genome sequences of Archaea are publicly available (b) the monophyletic nature of the Archaea is under discussion [41] and most important (c) joint data set of eubacterial and archaeal sequences produce less reliable alignments due to differences in ribosome composition between both domains [42].

In summary, RibAlignDB provides a good starting point for scientists who are interested in phylogenetic reconstructions based on concatenated ribosomal protein sequence. Sequences from future genome sequences can be added relatively easy due to RibAlign's powerful import filters.

\section{Computational requirements}

Phylogenetic reconstructions based on large alignments are very hardware-demanding, especially when likelihood-based methods are used in conjunction with resampling techniques. Even with the few available genomes today, concatenated alignments of ribosomal proteins sequences can easily exceed one million individual positions. Therefore, a selection in species and sequences has to be made for the more CPU-intensive treeing methods.

The MrBayes ${ }^{1}$ phylogenetic reconstruction program is fast since it is optimized for speed. However, this speed comes at the price of high memory requirements. As an example, a tree for 120 species and 5182 amino acid positions was calculated within a few days on a dual 3.0 GHz Xeon machine, but the calculation required 8 GB of main memory even when only two chains were used (tree not shown). Thus, larger data sets require either more memory or an MPI-aware cluster running the MPI-version of MrBayes.

The PHYLIP package ${ }^{2}$ is comprised of programs for different kinds of phylogenetic analysis. For a bootstrapped maximum-likelihood tree with ProML, raw computing power is the limiting factor. As an example (Figure 1), a ProML run with 100 replicates for the above-mentioned data set (120 species with 5182 amino-acid positions each) took more than three months to compute on a tennode cluster of dual $2.8 \mathrm{GHz}$ Xeon processors (with each node calculating ten trees). Future improvements in processor speed and the growing use of cluster computing in bioinformatics will hopefully keep up with the increasing computational demand. However, since the computational requirements increase progressively with alignment size and the number of species, a situation like with today's 16S rRNA phylogeny is likely, where a de novo tree based on all available sequences cannot be computed any more.

\section{Tree topology}

In the above-mentioned maximum likelihood tree calculated from concatenated ribosomal protein subunit sequences, all major phyla are well resolved (Figure 1). The topology is in good agreement with the widely accepted 16S rRNA-derived topology and also with a recently published tree based on concatenated ribosomal proteins subunit sequences [23].

The corresponding MrBayes tree showed the same topology (data not shown). Posterior probabilities computed from 13,000 trees showed good support for several of the earlier proposed super-clades, namely affiliation of Actinobacteria and Cyanobacteria [4], of Chlamydiae and Planctomycetes [20], and of Chlorobi and Bacteroidetes [43]. However, good statistical node support does not preclude tree reconstruction artifacts [44]. For example, different evolutionary rates might lead to artificial clustering of fast-evolving species due to long branch attraction. In addition, a common thermophilic lifestyle like that of Aquifex aeolicus VF5 and Thermotoga maritima $\mathrm{MSB}^{\mathrm{T}}$ is likely to impose similar constraints on amino acid composition and thus could cause an artificial clustering of these organisms. There are indeed indications that support an affiliation of Aquifex aeolicus VF5 with the Proteobacteria rather than with Thermotoga maritima MSB8 ${ }^{\mathrm{T}}$ [45]. Likewise, the association of the Actinobacteria and Cyanobacteria might be influenced by a biased amino acid composition as well [21].

A more in-depth discussion of the tree topology is beyond the scope of this paper. A much more detailed version of the ProML tree, showing all 120 species, can be obtained from the RibAlign website [39].

\section{Applicability}

The applicability of phylogenetic reconstructions based on concatenated ribosomal proteins sequences has been discussed elsewhere in detail [20]. As with all proteinbased phylogenies, concatenation of protein sequences has to face the problems of LGT and paralogy. LGT has been reported for some of the ribosomal protein encoding genes $[46,47]$ and others do not qualify as makers because they have paralogs or are not universally present in all eubacteria. In addition, individual proteins in a concatenated alignment might evolve at different speeds, which requires the applications of more sophisticated likelihood-based models to account for this type of sequence heterogeneity [48]. Finally, site selection can have an impact on the positions of weakly supported branches of the inferred trees $[20,25]$. 
To be fair, most of these problems apply to the 16S rRNA approach as well. LGT of 16S rRNA genes is possible [49] and has been reported $[50,51]$. In addition, most bacteria have paralogs of the $16 \mathrm{~S}$ rRNA gene that can differ considerably [52]. Also site selection has a major impact on the tree topology of 16S rRNA-based trees as well [6].

In the end, all trees that have been published so far based on concatenated ribosomal protein sequences are remarkably similar and mostly agree with the currently accepted $16 \mathrm{~S}$ rRNA-based tree topology.

\section{Conclusion}

Since the genomic revolution started in 1995 with the complete sequencing of Haemophilus influenzae Rd KW20 [53], new genomes are being sequenced at an exponentially increasing rate. This enables for new approaches in bacterial phylogeny that try to exploit a larger proportion from the genomic information for tree reconstruction than just single marker genes. To use such methods in an effective manner, a specialized and curated database of all potential marker genes from all genomes would be desirable.

RibAlign is a step in this direction for eubacterial ribosomal protein subunit sequences. We hope that it will be a helpful tool for scientists involved in whole genome sequencing of Eubacteria, particularly with regard to the phylogeny of representatives of new or only sparsely studied phyla.

\section{Availability and requirements \\ - Project name: RibAlign}

- Project home page: http://www.megx.net/ribalign

- Operating system(s): Mac OS X

- Programming language: REALbasic front end on top of a Valentina object-relational database

- Other requirements: none

- License: license-free

- Any restrictions to use by non-academics: RibAlign may not be sold or bundled with any type of commercial application

\section{List of abbreviations}

LGT - lateral gene transfer

megx - marine environmental genomics

MPI - message passing interface
PHYLIP - phylogeny inference package

RDP - ribosomal database project

rRNA - ribosomal ribonucleotide acid

\section{Authors' contributions}

RibAlign was implemented by HT. FOG contributed important ideas regarding features, implementation, tested the program and was involved in the writing of the manuscript.

\section{Note}

${ }^{1}$ MrBayes v 3.0B4 was used - version 3.1, which came out after our analysis, has lower memory requirements

${ }^{2}$ PHYLIP v. $3.6 a 4$ was used

\section{Acknowledgements}

We thank the Max Planck Society for supporting this work and Marisano James for spell-checking the manuscript and the RibAlign website.

\section{References}

I. Cole JR, Chai B, Farris RJ, Wang Q, Kulam SA, McGarrell DM, Garrity GM, Tiedje JM: The Ribosomal Database Project (RDP-II): sequences and tools for high-throughput rRNA analysis. Nucleic Acids Res 2005, 33(Database issue):D294-6.

2. Ludwig W, Strunk O, Westram R, Richter L, Meier H, Yadhukumar, Buchner A, Lai T, Steppi S, Jobb G, Forster W, Brettske I, Gerber S, Ginhart AW, Gross O, Grumann S, Hermann S, Jost R, Konig A, Liss T, Lussmann R, May M, Nonhoff B, Reichel B, Strehlow R, Stamatakis A, Stuckmann N, Vilbig A, Lenke M, Ludwig T, Bode A, Schleifer KH: ARB: a software environment for sequence data. Nucleic Acids Res 2004, 32(4): | 363-|37|.

3. Ludwig W, Strunk $O$, Klugbauer $S$, Klugbauer $N$, Weizenegger $M$, Neumaier J, Bachleitner M, Schleifer KH: Bacterial phylogeny based on comparative sequence analysis. Electrophoresis 1998 , 19(4):554-568.

4. Wolf YI, Rogozin IB, Grishin NV, Tatusov RL, Koonin EV: Genome trees constructed using five different approaches suggest new major bacterial clades. BMC Evol Biol 200I, I(I):8.

5. Wolf YI, Rogozin IB, Grishin NV, Koonin EV: Genome trees and the tree of life. Trends Genet 2002, 18(9):472-479.

6. Brochier C, Philippe H: Phylogeny: a non-hyperthermophilic ancestor for bacteria. Nature 2002, $417(6886): 244$.

7. $\mathrm{Di}$ Giulio $\mathrm{M}$ : The ancestor of the Bacteria domain was a hyperthermophile. J Theor Biol 2003, 224(3):277-283.

8. Nesbo CL, Boucher Y, Doolittle WF: Defining the core of nontransferable prokaryotic genes: the euryarchaeal core. J Mol Evol 200I, 53(4-5):340-350.

9. Zhaxybayeva O, Gogarten JP: Bootstrap, Bayesian probability and maximum likelihood mapping: exploring new tools for comparative genome analyses. BMC Genomics 2002, 3(I):4.

10. Woese CR: Interpreting the universal phylogenetic tree. Proc Natl Acad Sci U S A 2000, 97( I 5):8392-8396.

II. Daubin V, Gouy M, Perriere G: Bacterial molecular phylogeny using supertree approach. Genome Inform Ser Workshop Genome Inform 200I, I 2:155-I64.

12. Daubin V, Gouy M, Perriere G: A phylogenomic approach to bacterial phylogeny: evidence of a core of genes sharing a common history. Genome Res 2002, I 2(7): 1080-1090.

13. Clarke GD, Beiko RG, Ragan MA, Charlebois RL: Inferring genome trees by using a filter to eliminate phylogenetically discordant sequences and a distance matrix based on mean normalized BLASTP scores. J Bacteriol 2002, 184(8):2072-2080.

14. Snel B, Bork P, Huynen MA: Genome phylogeny based on gene content. Nat Genet 1999, 21(1): 108-110. 
15. Korbel JO, Snel B, Huynen MA, Bork P: SHOT: a web server for the construction of genome phylogenies. Trends Genet 2002 , I 8(3): | $58-162$.

16. Tekaia $F$, Lazcano A, Dujon B: The genomic tree as revealed from whole proteome comparisons. Genome Res 1999, 9(6):550-557.

17. Yang S, Doolittle RF, Bourne PE: Phylogeny determined by protein domain content. Proc Natl Acad Sci U S A 2005, I 02(2):373-378

18. Lin J, Gerstein M: Whole-genome trees based on the occurrence of folds and orthologs: implications for comparing genomes on different levels. Genome Res 2000, 10(6):808-818.

19. Pride DT, Meinersmann RJ, Wassenaar TM, Blaser MJ: Evolutionary implications of microbial genome tetranucleotide frequency biases. Genome Res 2003, I3(2): |45-I58.

20. Teeling H, Lombardot T, Bauer M, Ludwig W, Glockner FO: Evaluation of the phylogenetic position of the planctomycete 'Rhodopirellula baltica' SH I by means of concatenated ribosomal protein sequences, DNA-directed RNA polymerase subunit sequences and whole genome trees. Int I Syst Evol Microbiol 2004, 54(Pt 3):79I-80 I.

21. Brochier C, Bapteste E, Moreira D, Philippe H: Eubacterial phylogeny based on translational apparatus proteins. Trends Genet 2002, I 8(I): I-5.

22. Matte-Tailliez O, Brochier C, Forterre P, Philippe H: Archaeal phylogeny based on ribosomal proteins. Mol Biol Evol 2002, I9(5):63I-639.

23. lyer LM, Koonin EV, Aravind L: Evolution of bacterial RNA polymerase: implications for large-scale bacterial phylogeny, domain accretion, and horizontal gene transfer. Gene 2004, 335:73-88.

24. Brochier C, Forterre P, Gribaldo S: Archaeal phylogeny based on proteins of the transcription and translation machineries: tackling the Methanopyrus kandleri paradox. Genome Biol 2004, 5(3):RI7.

25. Hansmann S, Martin W: Phylogeny of 33 ribosomal and six other proteins encoded in an ancient gene cluster that is conserved across prokaryotic genomes: influence of excluding poorly alignable sites from analysis. Int J Syst Evol Microbiol 2000, 50 Pt 4: 1655-1663.

26. Brown JR, Douady CJ, Italia MJ, Marshall WE, Stanhope MJ: Universal trees based on large combined protein sequence data sets. Nat Genet 200I, 28(3):28I-285.

27. Brochier C, Gribaldo S, Zivanovic Y, Confalonieri F, Forterre P. Nanoarchaea: representatives of a novel archaeal phylum or a fast-evolving euryarchaeal lineage related to Thermococcales? Genome Biol 2005, 6(5):R42.

28. Daubin V, Moran NA, Ochman H: Phylogenetics and the cohesion of bacterial genomes. Science 2003, 301 (5634):829-832.

29. Jain R, Rivera MC, Lake JA: Horizontal gene transfer among genomes: the complexity hypothesis. Proc Natl Acad Sci U S A 1999, 96(7):380|-3806.

30. Harris JK, Kelley ST, Spiegelman GB, Pace NR: The genetic core of the universal ancestor. Genome Res 2003, I3(3):407-4I 2.

31. Gribaldo S, Philippe H: Ancient phylogenetic relationships. Theor Popul Biol 2002, 6 I (4):391 -408.

32. REAL Software Inc. homepage [http://www.realsoftware.com]

33. Paradigma Software, Inc. homepage.

34. Felsenstein J: PHYLIP (Phylogeny Inference Package), version 3.6. Distributed by the author Department of Genome Sciences, University of Washington, Seattle 2004.

35. Huelsenbeck JP, Ronquist F: MRBAYES: Bayesian inference of phylogenetic trees. Bioinformatics 200I, I 7(8):754-755.

36. Ronquist F, Huelsenbeck JP: MrBayes 3: Bayesian phylogenetic inference under mixed models. Bioinformatics 2003 I9(12): I572-1574.

37. Katoh K, Kuma K, Toh H, Miyata T: MAFFT version 5: improvement in accuracy of multiple sequence alignment. Nucleic Acids Res 2005, 33(2):51 I-5 I8.

38. MAFFT homepage [http://www.biophys.kyoto-u.ac.jp/ katoh/ programs/align/mafft/]

39. RibAlign homepage [http://www.megx.net/ribalign]

40. Apweiler R, Attwood TK, Bairoch A, Bateman A, Birney E, Biswas M, Bucher P, Cerutti L, Corpet F, Croning MD, Durbin R, Falquet L, Fleischmann W, Gouzy J, Hermjakob H, Hulo N, Jonassen I, Kahn D, Kanapin A, Karavidopoulou Y, Lopez R, Marx B, Mulder NJ, Oinn TM,
Pagni M, Servant F, Sigrist C], Zdobnov EM: The InterPro database, an integrated documentation resource for protein families, domains and functional sites. Nucleic Acids Res 200I, 29(I):37-40.

41. Cammarano P, Creti R, Sanangelantoni AM, Palm P: The archaea monophyly issue: A phylogeny of translational elongation factor $\mathbf{G ( 2 )}$ sequences inferred from an optimized selection of alignment positions. J Mol Evol I999, 49(4):524-537.

42. Lecompte O, Ripp R, Thierry JC, Moras D, Poch O: Comparative analysis of ribosomal proteins in complete genomes: an example of reductive evolution at the domain scale. Nucleic Acids Res 2002, 30(24):5382-5390.

43. Gupta RS: The phylogeny and signature sequences characteristics of Fibrobacteres, Chlorobi, and Bacteroidetes. Crit Rev Microbiol 2004, 30(2): I 23-I43.

44. Delsuc F, Brinkmann $H$, Philippe $H$ : Phylogenomics and the reconstruction of the tree of life. Nat Rev Genet 2005 6(5):36 I-375

45. Philippe $\mathrm{H}$, Laurent J: How good are deep phylogenetic trees? Curr Opin Genet Dev 1998, 8(6):616-623.

46. Brochier C, Philippe H, Moreira D: The evolutionary history of ribosomal protein RpSI4: horizontal gene transfer at the heart of the ribosome. Trends Genet 2000, I 6( I 2):529-533.

47. Garcia-Vallve S, Simo FX, Montero MA, Arola L, Romeu A: Simultaneous horizontal gene transfer of a gene coding for ribosomal protein 127 and operational genes in Arthrobacter sp. J Mol Evol 2002, 55(6):632-637

48. Yang Z: Maximum-Likelihood Models for Combined Analyses of Multiple Sequence Data. J Mol Evol I 996, 42(5):587-596.

49. Asai T, Zaporojets D, Squires C, Squires CL: An Escherichia coli strain with all chromosomal rRNA operons inactivated: complete exchange of rRNA genes between bacteria. Proc Natl Acad Sci U S A 1999, 96(5): 1971-1976.

50. Yap WH, Zhang Z, Wang Y: Distinct types of rRNA operons exist in the genome of the actinomycete Thermomonospora chromogena and evidence for horizontal transfer of an entire rRNA operon. | Bacteriol | 999, I 8 I ( I 7):520|-5209.

5I. Schouls LM, Schot CS, Jacobs JA: Horizontal transfer of segments of the 16S rRNA genes between species of the Streptococcus anginosus group. J Bacteriol 2003, I 85(24):724I-7246.

52. Marchandin H, Teyssier C, Simeon De Buochberg M, Jean-Pierre H, Carriere C, Jumas-Bilak E: Intra-chromosomal heterogeneity between the four I6S rRNA gene copies in the genus Veillonella: implications for phylogeny and taxonomy. Microbiology 2003, |49(Pt 6): |493-|50|

53. Fleischmann RD, Adams MD, White O, Clayton RA, Kirkness EF, Kerlavage AR, Bult CJ, Tomb JF, Dougherty BA, Merrick JM, et al: Whole-genome random sequencing and assembly of Haemophilus influenzae Rd. Science I995, 269(5223):496-5I 2

Publish with Biomed Central and every scientist can read your work free of charge

"BioMed Central will be the most significant development for disseminating the results of biomedical research in our lifetime. "

Sir Paul Nurse, Cancer Research UK

Your research papers will be:

- available free of charge to the entire biomedical community

- peer reviewed and published immediately upon acceptance

- cited in PubMed and archived on PubMed Central

- yours - you keep the copyright
BioMedcentral 